\title{
Preface to Volume 19
}

\section{Essential Metals in Medicine: Therapeutic Use and Toxicity of Metal Ions in the Clinic}

The metal ions discussed in this volume play significant roles in medicine. Most are deemed 'essential' for life: iron, manganese, cobalt, zinc, copper, molybdenum, and the metalloid selenium. The essentiality of others, such as chromium, vanadium, and nickel, remains the subject of intense debate. As pointed out in Chapter 1, whether essential (or not), maintenance of metal ion homeostasis in humans is crucial, as perturbations often result in disease. In addition to the (possibly) essential metal ions, other metal ions play important roles in human health, often by causing harm (e.g., the metalloid arsenic) but also by their use in the diagnosis or treatment of human diseases (gadolinium, gallium, cobalt, molybdenum, lithium, gold, silver).

After going through a brief history of drug development in Chapter 2, readers may appreciate the continuing challenges faced in human medicine. While the rise of biopharmaceuticals has proven beneficial for numerous diseases, they are costly, and unlikely to provide 'cures' for all diseases. Thus, small molecules continue to play an important role in the treatment of human diseases, as outlined in the discussion of attempts to solve two therapeutic challenges: malaria and Alzheimer's disease.

Iron, while arguably the most intensely studied metal ion in human medicine, remains poorly understood. Iron is essential for life, yet toxic, as elegantly presented in Chapters 3 to 7 . The essentiality of iron for both humans and pathogens means that the administration of iron is a double-edged sword. As discussed in Chapter 3, excess iron can prove toxic, and removal, via chelation therapy, remains a widely underappreciated problem in many countries where thalassemia is common and the availability of safe, inexpensive, and orally available iron chelators is desperately needed. Disruptions in iron homeostasis are clinically

Metal Ions in Life Sciences, Volume 19 Guest Editor: Peggy L. Carver

Series Editors: Astrid Sigel, Eva Freisinger, and Roland K. O. Sigel

(C) Walter de Gruyter GmbH, Berlin, Germany 2019, www.mils-WdG.com https://doi.org/10.1515/9783110527872-202 
problematic. In Chapter 4, the role of iron in several common neurodegenerative diseases is reviewed, as well as novel therapeutic measures aimed at amelioration of these conditions by removal of iron from the brain.

In Chapter 5, the controversy over whether iron administration increases the risk of infections, by providing 'food' for pathogens seeking to infect humans, outweighs its risk, is reviewed. In patients in whom iron administration is needed, controversy also exists regarding the optimal formulation of intravenous iron. As noted in Chapter 6, intravenous iron products may have a varying ability to release labile iron into plasma, leading to induction of the formation of highly reactive free radicals. On the other end of the spectrum, Chapter 7 discusses progress towards a long-held desire of clinicians to hijack the siderophore methodology commonly utilized by pathogens to scavenge iron as a means of delivering antimicrobials to those same pathogens, in a 'Trojan horse' approach to drug delivery. Despite many decades of work, this as-yet elusive dream has proved much more difficult than initially believed. Nevertheless, the first agents approved for human use may soon be available, if any of several agents that are currently undergoing clinical trials in humans prove successful.

Vanadium has had a long and storied history in medicine, with numerous claims of its beneficial effects on human health. Indeed, for many years, a variety of vanadium compounds were vigorously pursued as potential antidiabetic agents. In Chapter 8, the history of vanadium is reviewed: its long-time use as a 'health supplement', as a potential antidiabetic agent, and finally, recent realization of its potential as an adjunctive immunomodulatory agent for the treatment of cancer.

Like vanadium, the 'essentiality' of chromium in humans remains controversial, since in fact, the definition of 'essential' also remains a subject of debate. Chapter 9 provides a historical overview of research attempts to elucidate the nature and roles of chromium in human health. Importantly, it points out that an in-depth knowledge of differences in the actions of metal ion species is crucial to our understanding of their biological roles and potential toxicities in humans; indeed, chromate, $\mathrm{Cr}(\mathrm{VI})$, is toxic and a carcinogen. Finally, the chapter provides approaches for resolving controversies in chromium biology in humans.

Manganese, the subject of Chapter 10, plays a significant role in human health and disease, including its essentiality while also noting the potential for toxicity, including neurotoxicity, when present in excess. Exposure to manganese is due to many sources, including environmental ones. Finally, remediation methods for patients with excess levels is also indicated.

The role of cobalt in human health is mainly based upon its important role in cobalamin (vitamin $\mathrm{B}_{12}$ ), as has been extensively reviewed in a recent MILS text (Volume 13, Chapter 9). To date, the role of cobalt in human health has mainly been as a cause of contact dermatitis or (more rarely) as a cause of dilated cardiomyopathy in patients exposed to high levels from metal hip prostheses or from consumption of beer containing cobalt sulfate as a foam stabilizer. The radioactive isotope ${ }^{60} \mathrm{Co}$ is used to deliver radiation for cancer chemotherapy. Chapter 11 reviews the potential role of cobalt as antimicrobial, antiviral, and chemotherapeutic agent, and as a possible inhibitor of amyloid- $\beta(\mathrm{A} \beta)$ formation for the treatment of Alzheimer's disease. 
In addition to the essential roles copper plays in life, as discussed in Chapter 1 , copper may be utilized as a drug, with the lethality of copper excess being exploited as a means of killing pathogens. In Chapter 12, the potential role of copper as an adjunctive chemotherapeutic agent to reduce angiogenesis is explored.

Chagas' disease, human African trypanosomiasis and leishmaniosis comprise three common, but neglected tropical diseases in desperate need of effective, affordable therapeutic agents. While a number of metal-based antiparasitic agents have been developed, as yet, none has been approved for use in humans, due to the rigorous regulations guiding drug approvals worldwide. Chapter 13 outlines ongoing approaches to the development of these desperately needed agents.

While the biological functions of cyanide are not completely understood, its life-threatening toxicities are well recognized. Cyanide toxicity can occur due to active poisoning but, more commonly, from accidental sources such as fires. Outlining of the clinical aspects of cyanide in Chapter 14 provides an excellent overview of the mechanisms of human cyanide toxicity and the metal-containing antidotes (generally cobalt or molybdenum compounds) used in its treatment.

Whether or not they are 'essential' for life, metal ions play significant roles in medicine, as diagnostic and therapeutic agents. Disruptions in metal ion homeostasis, whether genetically induced, or from environmental exposure, are implicated in the pathogenesis of human diseases. Utilizing translational approaches to furthering our understanding of the chemical basis and human (biological) consequences of metal ion homeostasis provides an important foundation for improving human health and disease.

Peggy L. Carver 
